Bei früheren Untersuchungen hatte ich ${ }^{1}$ ) in Auszügen von Malagarosinen, welche in Rom gekauft worden waren, ziemlich genau Invertzucker angetroffen, wie aus nachstehenden Zahlen hervorgeht, in welchen die für die verlangte Drehung (Ventzke, $200 \mathrm{~mm}$-Rohr) angegebenen Werthe nach der folgenden Gleichung berechnet worden sind:

$$
27,418:-(42,4-1 / 2 \mathrm{t})=\mathrm{m}: \mathrm{x} \text {. }
$$

In letzterer bedeutet $\mathrm{m}$ den für $100 \mathrm{ccm}$ der Auszüge auf Invertzucker berechneten reducirenden Zucker.

\begin{tabular}{|c|c|c|c|c|}
\hline Rosinenart & $\begin{array}{l}\text { Proc. Zucker in } \\
\text { den Auszügen }\end{array}$ & $\begin{array}{l}\text { Polarisations- } \\
\text { temperatur }\end{array}$ & $\begin{array}{c}\text { Beobachtete } \\
\text { Drehung }\end{array}$ & $\begin{array}{l}\text { Verlangte } \\
\text { Drehung }\end{array}$ \\
\hline $\begin{array}{c}\text { Malaga I. } \\
-\end{array}$ & $\begin{array}{r}8,80 \\
15,61\end{array}$ & $\begin{array}{l}16,5^{0} \\
15,0^{0}\end{array}$ & $\begin{array}{r}-10,7 \\
-19,4\end{array}$ & $\begin{array}{r}11,0 \\
-19,9\end{array}$ \\
\hline
\end{tabular}

Dagegen hatte ich damals bei einem Auszuge von Zibibbo, welcher 9,63 Proc. reducirenden, als Invertzucker berechneten Zucker enthielt, eine Linksdrehung um 10,1 Theilstriche (Ventzke, $200 \mathrm{~mm}-\mathrm{Rohr} \mathrm{t}=20^{\circ}$ ) beobachtet, während jene Menge Invertzucker eine Rotation um - 11,4 verlangt haben würde. Die beobachtete Drehung entsprach nur 8,55 Proc. Zucker, während thatsächlich 9,63 Proc. gefunden worden waren. Hier ist somit mehr Dextrose als Lüvulose vorhanden gewesen, und zwar würden bei der Bezugnahme auf 20 Proc. Zucker 2,24 mehr direkt gefunden worden sein, als sich aus der Drehung berechneten.

E. Mach und K. Portele ${ }^{2}$ ) haben in 6 Mustern Rosinen von Malaga und Griechenland durchweg mehr Lävulose als Dextrose gefunden. Dagegen hatte $\mathrm{Mach}^{3}$ ) in Malagarosinen, welche über $1 \mathrm{Jahr}$ alt waren, etwas mehr Dextrose als Lävulose angetroffen, nämlich durch Titrirung 54,45 und durch Polarisation 52,7 Proc. Zucker gefunden. Er schloss daraus, dass bei langem Lagern von Trauben sich das Verhältmiss zwischen Dextrose und Lävulose wieder herzustellen scheine, wie es im Invertzucker vorliegt, oder dass sogar die Dextrose zum Vorherrschen gelangen könne. Aehnliches hatte bereits Dubrunfaut ${ }^{4}$ ) beobachtet, welcher von einer Zerstörung der Lävulose in den Rosinen durch Insektenfrass oder durch eine Art von Gährung sprach.

\title{
Ueber den spektroskopischen Nachweis der organischen Farbstoffe.
}

Von

J. Formánek, k. k. Inspektor.

Vorläufige Mittheilung aus der k. k. Lebensmittel-Untersuchungsanstalt der böhmischen Universität in Prag.

Es gelangen öfters gefärbte Gegenstände bezw. Lebensmittel zur Untersuchung, in denen entweder der Farbstoff als solcher festzustellen, oder eine

1) Zeitschr. angew. Chem. 1889, 538.

2) Weinlaube 1880, 545 .

3) Annalen der Oenologie 1876, 5, 421.

4) Compt. rend. 1849, 29, 51 . 
etwaige künstliche Färbung nachzuweisen ist. Manchmal ist es auch wünschenswerth, ja sogar nöthig, die Identität eines vorhandenen Farbstoffes mit einem anderen festzustellen.

In der Literatur findet man eine Reihe von ziemlich guten, aber komplicirten Methoden zur Bestimmung der Theer- und Pflanzenfarbstoffe; viele begnügen sich nun damit, blos die Anwesenheit oder Abwesenheit eines fremden Farbstoffes, den sauren oder basischen Charakter, höchstens die Gruppe eines eventuell vorhandenen Farbstoffes zu konstatiren. Systematische Methoden zur Bestimmung der einzelnen Farbstoffe, wie z. B. die von Weingärtner und Rota, welche auf der Einwirkung von Reduktionsmitteln auf verdünnte Farbenlösungen gegründet sind, sind zwar sehr gut, aber ziemlich komplicirt und die Methode von Rota erfordert ausserdem gute Kenntnisse der organischen Farbstoffe. Von praktischer Bedeutung ist auch die Ausfärbemethode; sie eignet sich zwar für viele Farbstoffe, aber sie reicht nicht immer zur Bestimmung mancher kombinirten Farbstoffe hin, und zwar aus dem Grunde, weil dieselben je nach Bedarf in verschiedenen Verhältnissen gemischt werden und die Ausfärbung des Gewebes infolgedessen immer verschieden ausfällt.

Diese Methoden, wie alle übrigen, sind jedoch nicht so empfindlich, dass man mit deren Hilfe alle einzelnen oder verwandten Farbstoffe oder Mischungen derselben auf eine leichte Art feststellen und unterscheiden kann, wie z. B. Malachitgrün von Chromgrün, Guineagrün B, Brillantgrün, Rhodamin G von Rhodamin O, Phloxin B von Phloxin BB, Safranin von Rhodulinroth, Methylviolett $1 \mathrm{~B}$ von Methylviolett $6 \mathrm{~B}$, Gemische von Eosin and Phloxin und viele andere.

Die Methode, welche, was die Empfindlichkeit und Genauigkeit anbelangt, alle bekannten Methoden übertrifft, ist die spektroskopische Methode. Das Spektroskop warde bisher seltener-benutzt und zwar nur zu einzelnen Untersuchungen; eine systematische spektroskopische Methode giebt es bis jetzt nicht.

Vogel empfahl in seinem Werke „Praktische Spektralanalyse irdischer Stoffe" die spektralanalytische Methode zur Untersuchung der Farbstoffe und beschrieb auch die Absorptionsspektra mehrerer Theer- und Pflanzenfarbstoffe. Vogel giebt aber die Lage der Absorptionsstreifen nur annähernd an und zwar auf Grund der Fraunhofer'schen Hauptlinien, was aber für die Beurtheilung aller Farbstoffe nicht genügt, sondern höchstens für einige charakteristische Farbstoffe oder einzelne Gruppen mehrerer Farbstoffe. Es wurde zwar von mehreren Seiten darauf hingewiesen, dass das Spektroskop für die Bestimmung der Farbstoffe am geeignetsten wäre, in vielen Büchern wurden auch manche Absorptionsspektren (nicht immer aber richtig) beschrieben und gezeichnet, aber ohne genaue Feststellung ihrer Lage und Eigenschaften; genaue Zeichnungen von Absorptionsspektren der organischen Farbstoffe sind ziemlich wenig veröffentlicht worden ${ }^{1}$ ). Wie bekannt, lassen die Lösungen der Farbstoffe je nach

1) Vergl. C. Grebe: Azofarben-Spektra u. A. 
ihren optischen Eigenschaften nur Lichtstrahlen der bestimmten Wellenlängen durch; beobachten wir nun solche Lösungen mittelst eines mit homogenem Lichte beleuchteten Spektroskopes, so bemerken wir, dass das Spektrum durch besondere, typische, einzelne oder mehrere dunkle Streifen unterbrochen ist, nach deren Beschaffenheit und Lage im Spektrum auf einen bestimmten Farbstoff geschlossen werden kann.

Diese einfache und bequeme Art des Nachweises der Farbstoffe veranlasste mich, diese Absorptionsspektren näher zu studiren. Bei diesem Studium bemerkte ich, dass die Lösungen vieler Farbstoffe gleiche Form der Absorptionsstreifen ausweisen, deren Lagen im Spektrum so nahe aneinander sich befanden, dass man sie blos, wie schon oben gesagt wurde, auf Grund der Fraunhofer' schen Hauptlinien nicht bestimmen kann. Deswegen habe ich die Dunkelheitsmaxima solcher Streifen mittelst des Spektrometers festgestellt und gefunden, dass verschiedene Farbstoffe auch verschiedene Lage der Absorptionsstreifen besitzen, so dass man die Natur der Farbstoffe grösstentheils schon auf Grund der genauen Feststellung der Lage ihrer Absorptionsstreifen bestimmen kann.

Bei Beobachtung verschiedener Absorptionsspektra habe ich gefunden:

1. dass die Streifen einiger Farbstoffe in gleichen Lösungsmitteln so nahe aneinander liegen, dass man sehr genau messen muss, um überhaupt die Differenz der Lagen festzustellen,

2. dass einige Farbstoffe entweder zum Messen unbrauchbare oder keine Absorptionsstreifen liefern, und

3. dass die Absorptionsstreifen einiger verschiedener Farbstoffe sich bei kleinerer Dispersion fast decken können.

Um diesen Mängeln abzuhelfen, beobachtete ich solche Farbstoffe in verschiedenen Lösungsmitteln und fand, dass z. B. die Farbstoffe, deren Absorptionsstreifen in wässerigen Lösungen sich stark näherten, in Aethyl- oder Amylalkohol wieder stark differirten. Die Farbstoffe, welche unbrauchbare Spektra lieferten, versuchte ich in eine zur spektralanalytischen Beobachtung geeignete Form überzuführen, oder aber dieselben mittelst chemischer Agentien in Verbindung mit der spektralanalytischen Beobachtung zu unterscheiden, was bei fast allen Farbstoffen gelungen ist und wodurch somit obiger Mangel beseitigt wurde. Einige durch die Lage ihrer Absorptionsstreifen verwandte Farbstoffe verhalten sich gegen Säure oder Basen verschieden; manche ändern sich durch Einwirkung der Säure, andere wieder durch die Einwirkung der Alkalien; so z. B. die Lösung des Cyanin B, dessen Absorptionsstreifen sich dem des Türkisblau G nähert, wird mit Salpetersäure gelb und ändert sich mit Kalihydrat nicht, wogegen die Lösung des Türkisblau G nach Zusatz der Salpetersäure nicht geändert, aber durch die Einwirkung von Kaliumhydrat entfärbt wird. Manche Farbstoffe ändern die Farbe durch Einwirkung von Säure, andere wieder durch Einwirkung von Basen und liefern andere charakteristische Spektra; manche entfärben sich in saurer, andere wieder in alkalischer Lösung, z. B. die alkoholische Lösung des Eosins entfärbt sich mit Säure, die alkoholische Lösung des Rhodamins S 
dagegen mit Alkali; die wässerige Lösung des Naphtolgelbs entfärbt sich mit Säure, die wässerige Lösung des Anramins dagegen mit Alkali.

Farbstoffe, deren Lösungen keine Absorptionsstreifen ausweisen, ändern durch Zusatz von Säure oder Alkali die Farbe und liefern Absorptionsstreifen; so z. B. die wässrige Lösung des Metanilgelbs, welche blos einseitige Absorption im Blauen zeigt, färbt sich durch Einwirkung von Salpetersäure karminroth und liefert einen Absorptionsstreifen. Die alkoholische Lösung des Azosäuregelbs, welche ebenfalls nur einseitige Absorption im Blauen zeigt, färbt sich durch Einwirkung von Kaliumhydrat karminroth und liefert einen von dem erstgenannten Farbstoffe verschiedenen Absorptionsstreifen.

Finden sich in Mischungen die Streifen einiger Farbstoffe so nahe aneinander, dass sie fast zusammenfliessen, so kann man durch Zusatz eines Reagens einen oder anderen Streifen verschieben, oder einen Streifen zum Verschwinden bringen; z. B. die Lösung des Rhodamins und Fuchsins liefert die Absorptionsstreifen nahe aneinander; setzt man zu solcher Lösung Salpetersäure zu, so verschiebt sich der Fuchsinstreifen nach links und versehwindet allmählich, so dass man ihn noch messen kann, und der Rhodaminstreif bleibt zurück.

Ein Gemisch von Methylviolett und Neublau $R$ liefert in der wässerigen Lösung nahe aneinanderliegende Absorptionsstreifen; setzt man zu einer solchen Lösung Salpetersäure hinzu, so schiebt sich der Absorptionsstreif von Methylviolett nach links, der Absorptionsstreif des Neublau $\mathbf{R}$ bleibt unverändert, und man kann beide Streifen bequem messen.

Bei allen diesen Versuchen habe ich ebenfalls gefunden, dass bestimmte Gruppen der Farbstoffe auch bestimmte typische Formen besitzen, und zwar entweder einen Streifen, wie z. B. (Fig. 3): grüne und einige blaue Triphenylmethanfarbstoffe, oder (Fig. 4): einige rothe Azofarbstoffe, wie Bordeanx, Echtroth, oder neben einem starken Streifen einen schwachen Streifen rechts (Fig. 5): Pyronine

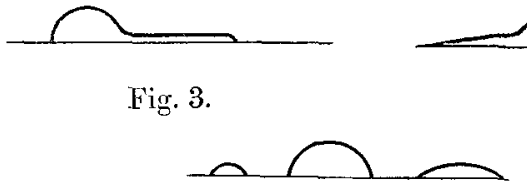

Fig. 6.

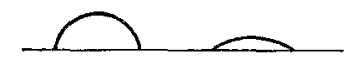

Fig. 5.

Fig. 4.

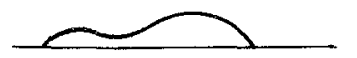

Fig. 7.

(Eosin, Erythrosin, Rhodamin), Safranine in Aethylalkohol, Fuchsine; oder neben einem starken Streifen zwei schwache Streifen zu beiden Seiten (Fig. 6): wie Phloxine, Phloxin B, BB, oder zwei wellenartig verbundene Streifen (Fig. 7) wie Azofarbstoffe: Ponceau, Azoeosin, oder auch mehrere Streifen, wie Alizaringrün $\mathrm{S}$, Cölestinblau B u. s. w., kurz gesagt, jede Gruppe der Farbstoffe zeigt eine charakteristische Form.

Ordnet man die Lösungen der Farbstoffe nebeneinander, wie ihre Absorptionsstreifen im Spektrum von der linken zur rechten Seite hintereinander folgen, so findet man, dass dieselben eine harmonische Skala bilden, d. i. die Farbe des Spektralfeldes, in welchem sich der Absorptionsstreifen findet, ergänzt sich mit der 
Farbe des Farbstoffes. Die Absorptionsstreifen finden sich also z. B. bei grünen Farbstoffen im rothen Felde und bei rothen Farbstoffen im grünen Felde des Spektrums. Von dieser Regel machen einige Farbstoffe eine Ausnahme, aber nur solche, deren Farbenton in eine andere Farbe übergeht, z. B. Methylenblau, Patentblau springen unter grüne Farbstoffe, Rosa Magdala unter violette Farbstoffe über u. s. w. Meistens aber verhalten sich die Farbstoffe so, dass man nach der Art des Farbstoffes schon im Voraus die ungefähre Lage seines Absorptionsstreifens im Spektrum bestimmen kann.

Auf Grund aller dieser Beobachtungen bearbeitete ich eine spektroskopische Methode zur Bestimmung der Farbstoffe, deren Princip folgendes ist:

Alle Farbstoffe theilte ich je nach der Form ihrer Absorptionsstreifen in Gruppen and zwar die rothen und die blauen Farbstoffe in je 7 Gruppen und die grünen und die gelben Farbstoffe in je 5 Gruppen.

Mittelst eines Spektroskopes von geeigneter Dispersion wird zuerst die Gruppe, in welche der gesuchte Farbstoff gehört, bestimmt, sodann wird mit Hilfe einer passenden Vorrichtung zum Messen die Lage des Absorptionsstreifens bestimmt; genügt die Bestimmung der Gruppe und der Lage nicht, so theilt man die Lösung des Farbstoffes in drei Theile; zu dem ersten setzt man Salpetersäure, zu dem zweiten Ammoniak und zu dem dritten Kalihydrat hinzu und beobachtet die Veränderung der Farbe und des Spektrums, und auf Grund dieser Beobachtungen sucht man den betreffenden Farbstoff in den zu diesem Zwecke zusammengestellten Tabellen aus.

Auf diese Art lassen sich alle diese Farbstoffe bestimmen, welche geeignete Absorptionsspektra liefern oder liefern können, oder aber auch solche, welche sich mit chemischen Reagentien ändern.

Im Nachfolgenden sei im Kurzen diese Methode näher beschrieben:

Der zur Bestimmung der Farbstoffe dienende Spektralapparat muss eine passende Dispersion besitzen; die Dispersion darf nicht $\mathrm{zu}$ gross sein, damit sich die Absorptionsstreifen nicht zu viel ausdehnen und dadurch eine genaue Messung unmöglich machen, und ausserdem auch, damit man die Uebersicht im Spektrum nicht verliert. Die Dispersion darf aber auch nicht zu klein sein, damit dic Streifen nicht zu nahe liegen. Weiter muss der Apparat eine geeignete Vorrichtung zum Messen besitzen. Diesen Bedingungen entsprechen die Apparate, welche das optische Institut von A. Krüss in Hamburg verfertigt. Die genannte Firma ist bereit, alle zu diesem Zwecke dienenden Apparate mit derselben Dispersion zu liefern, so dass die SpektraltafeIn, die ich herauszugeben beabsichtige, mit diesen Apparaten ohne jede Umrechnung benutzt werden können. Der Spektralapparat, den man wohl auch für gewöhnliche spektralanalytische Untersuchungen verwenden kann, hat die Form des Bunsen'schen Spektralapparates. Der Spalt ist symmetrisch, d. h. beide Hälften bewegen sich mittelst einer getheilten Trommel symmetrisch gegen die optische Achse des Apparates.

Die Messvorrichtung (Fig. 8) ist darauf eingerichtet, dass sich das mit einem festen und mit der Skala korrespondirenden Fadenkreuze versehene Fernrohr (a) mittclst einer Mikrometerschraube (b) um die vertikale Achse des Apparates 
bewegt. Ganze Umdrehungen werden mittelst eines Indexes (i) auf der unter dem Fernrohre befestigten Skala (c), theilweise Umdrehungen auf der mit der Mikrometerschraube verbundenen, in 100 Theile getheilten Trommel (d) abgelesen.

Die Skala ist zur leichteren Kontrolle des Apparates und um die Spektraltafeln zu vereinfachen, so eingerichtet, dass die Mitte beider D-Linien gerade auf 10,00 fällt. Zum Beleuchten eignet sich am besten die Auer'sche Lampc. Intensives Licht ist besonders zur Beobachtung im blauen und blauvioletten Theile des Spektrums nöthig, wo sich die Absorptionsstreifen der orangegelben und gelben Farbstoffe befinden, denn in Folge der schwächeren Absorption dieser Farbstoffe ist es nöthig, entweder stärkere Schichten oder etwas mehr koncentrirte Lösungen zu beobachten; ohnedies aber unter Benutzung eines intensiven Lichtes kann man stärkere Lösungen beobachten, in welchen die Streifen scharf auftreten und sich besser messen lassen. Bei Anwendung des Auer'schen Lichtes habe ich auch neue Absorptionsstreifen bei einigen Farbstoffen gefunden, so z. B. bei der wässe-

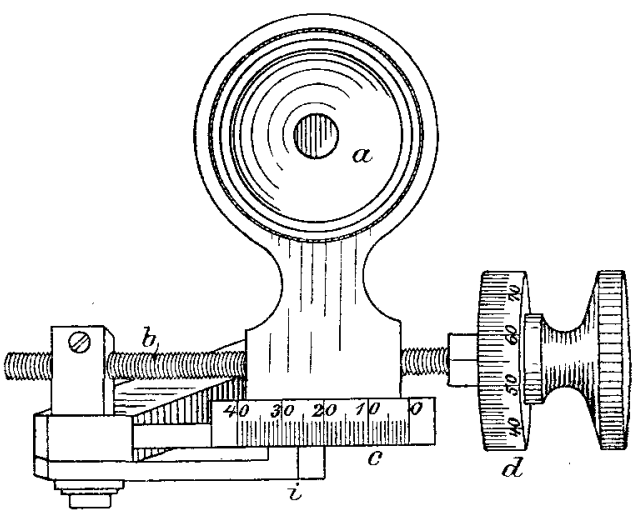

Fig. 8. rigen Lösung des Safrans, von welchem angeführt wird, dass er nur einseitige Absorption liefert, ebenfalls beim Phloxin, Fuchsin und einigen anderen Farbstoffen. Bei der Revision der bekannten Absorptionsspektra habe ich gefunden, dass manche Angaben und Zeichnungen unrichtig sind, so z. B. wird von Safranin angeführt, dass es einen Streifen liefert; in Wirklichkeit aber unter Benutzung eines scharfen Lichtes sieht man einen schwachen Doppelstreifen u. s. w.

Als Lösungsmittel verwende ich destillirtes Wasser, 97\%-igen Acthylalkohol und Amylalkohol. Den Amylalkohol halte ich für ein Hauptlösungsmittel, weil in demselben die Absorptionsstreifen am engsten und so scharf erscheinen, dass man sie ganz genau messen kann.

Alle Lösungsmittel müssen chemisch rein und neutral sein, da manche Absorptionsspektra sich schon durch eine Spur von Säure oder Alkali ändern; ebenfalls muss man bei Anwendung der Glaseprouvetten peinliche Reinlichkeit einhalten. Für Theerfarbstoffe verwende ich Salpetersäure 1 : 5, Ammoniak (spec. Gew. 0,96) 1:5, Kalihydrat in Wasser und Aethylalkohol im Verhältnisse 1:10. Zur Untersuchung der Pflanzenfarbstoffe verwende ich Schwefelsäure $1: 5$, Essigsäure $1: 5$ und Alaun $1: 12$.

Der zu untersuchende Farbstoff wird in Wassêr, Aethylalkohol und Amylalkohol gelöst, in eine gewöhnliche, dünnwandige Glaseprouvette gebracht und dicht vor den Spalt des beleuchteten Apparates gestellt.

Sind die Lösungen koncentrirt, so wird allmählich mit dem betreffenden Lösungsmittel verdünnt, bis der Absorptionsstreifen bezw. die Streifen scharf auftreten. 
Nach der Form des Absorptionsspektrums wird die Gruppe bestimmt. Manche Farbstoffe sind so charakteristisch, dass man sie auf den ersten Blick erkennen kann; hierbei sei an dieser Stelle bemerkt, dass, wenn zwei oder mehrere Streifen im Spektrum vorkommen, gewöhnlich nur ein Streif stark hervortritt, die übrigen dagegen sind schwächer und verschwinden durch stärkere Verdünnung. Will man daher die Form des Spektrums feststellen, so muss man allmähhlich verdünnen.

Durch die Bestimmung der Gruppe wird der gesuchte Farbstoff auf eine kleine Anzahl der Farbstoffe beschränkt. Nachher wird die Lösung des Farbstoffes so weit verdünnt, dass der Absorptionsstreifen bezw. die Streifen möglichst eng, dabei aber scharf und gut sichtbar erscheinen. Die Lage der Absorptionsstreifen ist nicht von der Koncentration der Lösung abhängig, denn das Dunkelheitsmaximum der Streifen bleibt, wie ich mich durch eine grosse Anzahl von Messungen überzeugt habe, bei jeder Koncentration konstant; natürlich fliessen in koncentrirten Lösungen, wenn das Absorptionsspektrum aus mehreren Streifen besteht, die Absorptionsstreifen zu einem breiten Streifen zusammen, man muss daher die Lösung so weit verdünnen, bis die Streifen scharf auftreten und durch weitere Verdünnung eben zu verschwinden anfangen. Die Temperaturunterschiede von $16-24^{\circ} \mathrm{C}$. haben auf die Lage der Absorptionsstreifen keinen merkbaren Einfluss.

Zur: Bestimmung des Farbstoffes nach dieser Methode ist aber gewöhnlich der Hauptstreifen maassgebend.

Nun wird die Lage des Absorptionsstreifens bestimmt. Es geschieht dies, indem man mittelst der Mikrometerschraube das Fernrohr bewegt, die Mitte des Fadenkreuzes genau auf die dunkelste Stelle des Streifens stellt und auf der Skala und Trommel abliest. Wenn man die Lage des Absorptionsstreifens festgestellt hat, so sucht man in den Tabellen auf, welcher Zahlenwerth der x-ten Gruppe mit dem gefundenen Werthe des untersuchten Farbstoffes übereinstimmt. Genügt die Bestimmung der Gruppe und der Lage der Absorptionsstreifen nicht, so theilt man die Lösung in drei Theile in die Eprouvetten, setzt in die erste Eprouvette drei Tropfen nach Vorschrift verdünnter Salpetersäure, in die zweite dieselbe Menge des Ammoniaks und in die dritte auch drei Tropfen Kalihydrat hinzu.

Gleichzeitig wird die Veränderung, welche durch Einwirkung des Reagens in der Lösung verursacht wird, beobachtet. Selbstverständlich wird auch jede Flüssigkeit, zu welcher Säure oder Alkali zugesetzt wurde, spektroskopisch beobachtet, falls sie sich nicht entfärbt.

Auf Grund der gemachten Beobachtungen und gewonnenen Resultate findet man in besonderen zu diesem Zwecke zusammengestellten Tabellen den gesuchten Farbstoff. Diese Tabellen sind so zusammengestellt, dass in denselben die Lage der Absorptionsstreifen in Wasser, in Aethylalkohol und in Amylalkohol angegeben wird und zwar in neutraler, saurer und alkalischer Lösung. Die Zahlen, welche die Lage der. Absorptionsstreifen im Spektrum angeben, beziehen sich theils auf die bestimmte Skala des Apparates, welche 
bei allen Krüss'schen Spektralapparaten beibehalten wird, theils auch auf bestimmte Wellenlängen, damit man diese Angaben auch auf die Skala anderer Apparate umrechnen kann. Die Zahlen und infolge dessen auch die Wellenlängenangaben sind womöglich von fünf $\mathrm{zu}$ fünf abgerundet. Gleichzeitig werden in diesen Tabellen alle Veränderungen, die in der Lösung nach Zusatz der Reagentien vor sich gehen, angezeigt. Diesen Tabellen werden ebenfalls genaue Zeichnungen der Spektra von verschiedenen Farbstoffen und ihre Umwandlungen beigegeben werden.

Zur Untersuchung von farbigen Gegenständen oder Farbstoffen werden verschiedene Lösungsmittel, je nach der Art des zu untersuchenden Gegenstandes, verwendet. Einige Stoffe, wie z. B. Zucker oder Dextrin, stören die spektroskopische Untersuchung nicht; man kann also die Zuckerwaare einfach in Wasser lösen.

Zur Trennung der kombinirten Farbstoffe verwende ich Amylalkohol und Aethylalkohol. Verschiedene Farbstoffe lösen sich in verschiedenen Lösungsmitteln. Der kombinirte Farbstoff wird zuerst mit Amylalkohol geschüttelt, der ungelöste Theil abfiltrirt, mit Aethylalkohol geschüttelt und am Ende mit Wasser behandelt. Die Lösungen werden spektroskopisch untersucht.

Kommt die wässerige Lösung eines Farbstoffes zur Untersuchung und genügt die Beobachtung dieser Lösung bei Anwendung der Reagentien nicht, so wird Amylalkohol zugesetzt und ausgeschüttelt; färbt sich der Amylalkohol nicht, so werden $3-4$ Tropfen Salpetersäure hinzugesetzt und darauf von Neuem ausgeschüttelt. In dem Falle wird der eventuell vorhandene und in Amylalkohol unlösliche Farbstoff aufgenommen. Z. B. Säurefuchsin löst sich in Amylalkohol nicht; wird aber Säure zugesetzt, so wird Säurefuchsin von Amylalkohol aufgenommen.

Ob die im Kurzen beschriebene Methode alle Fragen kombinirter Farbstoffe zu lösen im Stande ist, kann ich heute noch nicht mit Bestimmtheit sagen, so viel aber habe ich festgestellt, dass die beschriebene Methode, was einzelne Farbstoffe anbelangt, genau jede Gattung und Art der Farbstoffe aufzufinden im Stande und so empfindlich ist, dass man z. B. Methylviolett 1 B von Methylviolett $6 \mathrm{~B}$, Phloxin von Phloxin $\mathrm{B}$ und von Phloxin B B genau unterscheiden kann, ein Ergebniss, welches mit keiner Methode bisher erzielt wurde.

Diese Methode setzt keine genaue Kenntnisse der organischen Farbstoffe voraus, also kann sie jeder, auch mit den organischen Farbstoffen weniger vertraute Chemiker mit Erfolg verwenden.

Ursprünglich habe ich die spektrochemische Methode nur für die Farbstoffe, welche zur Färbung der Lebensmittel verwandt werden, ausgearbeitet; da ich aber die Bestimmung anderer Farbstoffe, besonders auch für Farbenchemiker wichtig halte, so nehme ich in die Tabellen alle wichtigsten Farbstoffe auf. Ich behalte es mir daher vor, mit der Zeit eine ausführliche Anleitung nebst Tabellen und genauen Zeichnungen der Spektren zu veröffentlichen.

Zum Schlusse mögen vier Beispiele der spektroskopischen Tabellen aufgeführt werden. 
Gruppe I.

Beispiel de]

\begin{tabular}{|c|c|c|c|c|c|c|}
\hline \multirow{2}{*}{ Handelsuame } & \multirow{2}{*}{ Farbe } & \multicolumn{4}{|c|}{ In Wasser } & \multirow[b]{2}{*}{ Absorptio } \\
\hline & & Absorption & Salpetersäure & $\begin{array}{c}\text { Ammo- } \\
\text { niak }\end{array}$ & $\begin{array}{c}\text { Kali- } \\
\text { hydrat }\end{array}$ & \\
\hline $\begin{array}{c}\text { Eosin I, gelblich } \\
{[\mathrm{By}]}\end{array}$ & $\begin{array}{l}\text { gelbroth } \\
\text { fluorescirt }\end{array}$ & $\begin{array}{r}\text { Hauptstreif } 13,60 \\
\left.[514]^{1}\right) \\
\text { Nebenstreif } 15,75 \\
{[482,25]}\end{array}$ & entfürbt sich & $\begin{array}{l}\text { ändert } \\
\text { sich nicht }\end{array}$ & $\begin{array}{l}\text { ändert } \\
\text { sich nicht }\end{array}$ & $\begin{array}{r}\text { Hauptstreif } 1 \\
\text { Nebenstreif } 1 \\
\text { [4? }\end{array}$ \\
\hline $\begin{array}{c}\text { Methyleosin } \\
{[A]}\end{array}$ & $\begin{array}{c}\text { gelbrotlı } \\
\text { fluorescirt }\end{array}$ & $\begin{array}{r}\text { Lamptstreif } 13,10 \\
{[522,5]} \\
\text { Nebenstreif } 15,40 \\
{[487]}\end{array}$ & entfärbt sich & $\begin{array}{l}\text { ändert } \\
\text { sich nicht }\end{array}$ & $\begin{array}{l}\text { ändert } \\
\text { sich nicht }\end{array}$ & $\begin{array}{r}\text { Hauptstreif } 1 \\
\text { Nebenstreif } 1 \\
{[4 !}\end{array}$ \\
\hline $\begin{array}{c}\text { Rhodamin } \\
{[\mathrm{B} y]}\end{array}$ & $\begin{array}{l}\text { rosaroth } \\
\text { fluorescirt }\end{array}$ & $\begin{array}{r}\text { Hanptstreif } 11,60 \\
{[551,5]} \\
\text { Nebenstreif } 13,55 \\
{[\overline{\mathbf{5}} \mathbf{5}]}\end{array}$ & $\begin{array}{c}\text { Farbe } \\
\text { unverändert } \\
\text { Hauptstreif } 11,45 \\
{[554,75,} \\
\text { Sebenstreif } 13,40 \\
{[517,5]}\end{array}$ & $\begin{array}{l}\text { ändert } \\
\text { sich nicht }\end{array}$ & $\begin{array}{l}\text { ändert } \\
\text { sich nicht }\end{array}$ & $\begin{array}{r}\text { Hauptstreif } 1 \\
{\left[54^{5}\right.} \\
\text { Nebenstreif } 1 \\
{[50}\end{array}$ \\
\hline $\begin{array}{c}\text { Rhodamin } 0 \\
{[\mathrm{M}]}\end{array}$ & $\begin{array}{l}\text { rosaroth } \\
\text { fluorescirt }\end{array}$ & $\begin{array}{r}\text { Hauptstreif } 11,45 \\
{[554,75]} \\
\text { Nebenstreif } 13,45 \\
{[516,5]}\end{array}$ & $\begin{array}{r}\text { Farbe } \\
\text { unverändert } \\
\text { Hauptstreif } 11,30 \\
{[558]} \\
\text { Nebenstreif } 13,30 \\
{[519]}\end{array}$ & $\begin{array}{l}\text { ändert } \\
\text { sich nicht }\end{array}$ & $\begin{array}{l}\text { ändert } \\
\text { sich nicht }\end{array}$ & $\begin{array}{r}\text { Hanptstreif } 1 \\
{[54 \%} \\
\text { Nebenstreif } 1 \\
{[50 t}\end{array}$ \\
\hline
\end{tabular}

\section{Gruppe II.}

Phloxin B

[D]

[L] flnorescirt

rosaroth fluorescint
Hauptstreif 13,50

$[515,75]$

Nebenstreife

$11,65 \quad 15,75$

$[550,5] \quad[482,25]$

Hauptstreif 13,35

$[518,25]$

Nebenstreife

11,70

15,55

$[519,25]$

[485] entfärbt sich

entfärht sich

ändert sich nicht sich nicht
Hauptstreif 1

Nebenstreif

$11,20 \quad 15$ $[560,25] \quad[48$ ؛

Hauptstreif 1

Nebenstreif

$11,20 \quad 15$

$[560,25] \quad[49 \%$

1) Wellenlängen. 
rothen Farbstoffe.

Gruppe I.

\begin{tabular}{|c|c|c|c|c|c|c|}
\hline \multicolumn{3}{|l|}{ ethylalkohol } & \multicolumn{4}{|c|}{ In Amylalkohol } \\
\hline alpetersäure & Ammoniak & $\begin{array}{l}\text { Kalihy* } \\
\text { drat in } \\
\text { Alkohol }\end{array}$ & Absorption & Salpetersäure & Ammoniak & $\begin{array}{l}\text { Kalihydrat } \\
\text { in Alkohol }\end{array}$ \\
\hline ntfärbt sich & \begin{tabular}{|} 
Farbe \\
unverändert \\
Hauptstreif 13,10 \\
{$[522,5]$} \\
Nebenstreif 15,45 \\
{$[486,5]$}
\end{tabular} & $\begin{array}{l}\text { wie bei } \\
\text { Am- } \\
\text { mo- } \\
\text { niak }\end{array}$ & $\begin{array}{r}\text { Hauptstreif } 12,45 \\
{[534,5]} \\
\text { Nebenstreif } 14,70 \\
{[497]}\end{array}$ & entfürbt sich & \begin{tabular}{|c|} 
Farbe \\
unverändert \\
Hauptstreif 12,95 \\
{$[525,25]$} \\
Nebenstreif 15,15 \\
{$[490,5]$}
\end{tabular} & $\begin{array}{l}\text { wie bei } \\
\text { Ammoniak }\end{array}$ \\
\hline ntfärbt sich & $\begin{array}{c}\text { Farbe } \\
\text { unverändert } \\
\text { Hauptstreif } 12,80 \\
{[528]} \\
\text { Nebenstreif } 15,15 \\
{[490,5]}\end{array}$ & $\begin{array}{c}\text { wie bei } \\
\text { Am- } \\
\text { mo- } \\
\text { niak }\end{array}$ & $\begin{array}{r}\text { Hauptstreif } \mathbf{1 2 , 1 5} \\
{[\mathbf{0 4 0 , 2 5 ]}} \\
\text { Nebenstreif } \mathbf{1 4 , 5 5} \\
{[498,25]}\end{array}$ & entfärbt sich & \begin{tabular}{|c|} 
Farbe \\
unverändert \\
Hauptstreif 12,50 \\
{$[533,5]$} \\
Nebenstreif 14,85 \\
{$[494,75]$}
\end{tabular} & $\begin{array}{c}\text { wie bei } \\
\text { Ammoniak }\end{array}$ \\
\hline $\begin{array}{c}\text { Farbe } \\
\text { unverändert } \\
\text { uptstreif } 11,60 \\
{[551,5]} \\
\text { benstreif } 13,65 \\
{[513,25]}\end{array}$ & andert sich nicht & $\begin{array}{l}\text { ändert } \\
\text { sich } \\
\text { nicht }\end{array}$ & $\begin{array}{r}\text { Hauptstreif } 11,85 \\
{[546,25]} \\
\text { Nebenstreif } 13,90 \\
{[509]}\end{array}$ & $\begin{array}{c}\text { Farbe } \\
\text { unverändert } \\
\text { Hauptstreif } 11,60 \\
{[551,5]} \\
\text { Nebenstreif } 13,65 \\
{[513,25]}\end{array}$ & \begin{tabular}{|c|} 
Farbe \\
unverändert \\
Hauptstreif 12,15 \\
{$[540,25]$} \\
Nebenstreif 14,40 \\
{$[501,5]$}
\end{tabular} & \begin{tabular}{|c} 
Farbe \\
unverändert \\
Hauptstreif 12,05 \\
{$[542,25]$} \\
Nebenstreif 14,20 \\
{$[504,5]$}
\end{tabular} \\
\hline $\begin{array}{c}\text { Farbe } \\
\text { unverändert } \\
\text { uptstreif } 11,45 \\
{[554,75]} \\
\text { benstreif } 13,55 \\
{[515]}\end{array}$ & ändert sich nicht & $\begin{array}{c}\text { ändert } \\
\text { sich } \\
\text { nicht }\end{array}$ & $\begin{array}{r}\text { Hauptstreif } 11,65 \\
{[550,5]} \\
\text { Nebenstreif } 13,70 \\
{[512,5]}\end{array}$ & \begin{tabular}{|c} 
Farbe \\
unverändert \\
Hauptstreif 11,45 \\
{$[554,75]$} \\
Nebenstreif 13,55 \\
{$[515]$}
\end{tabular} & \begin{tabular}{|c|} 
Farbe \\
unverändert \\
Hauptstreif 11,95 \\
{$[544,25]$} \\
Nebenstreif 14,05 \\
{$[506,75]$}
\end{tabular} & $\begin{array}{c}\text { wie bei } \\
\text { Ammoniak }\end{array}$ \\
\hline
\end{tabular}

Gruppe II.

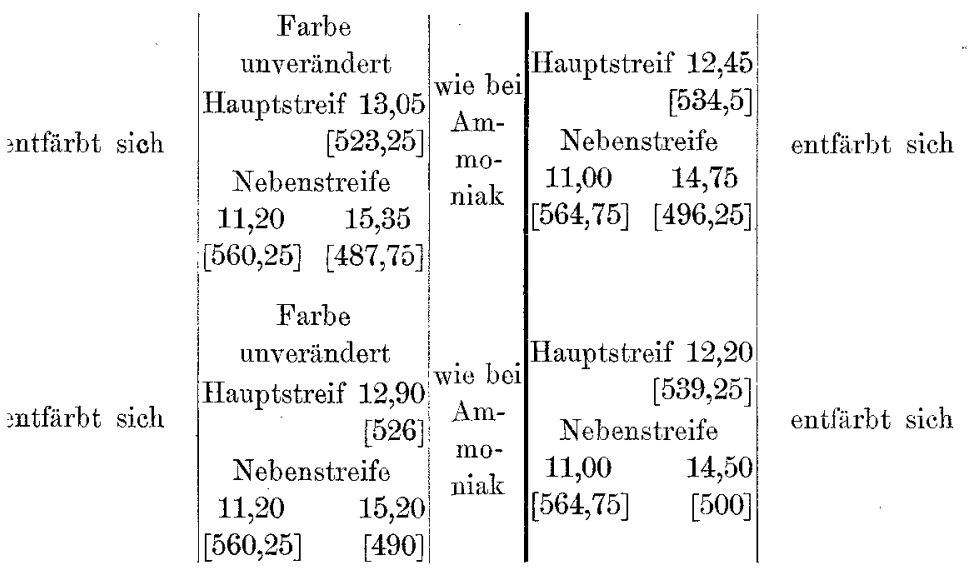

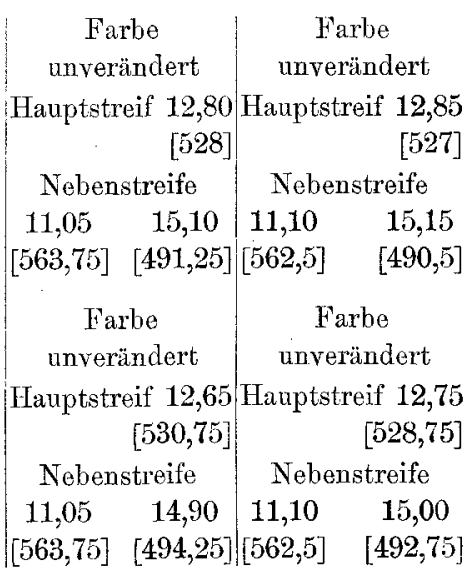




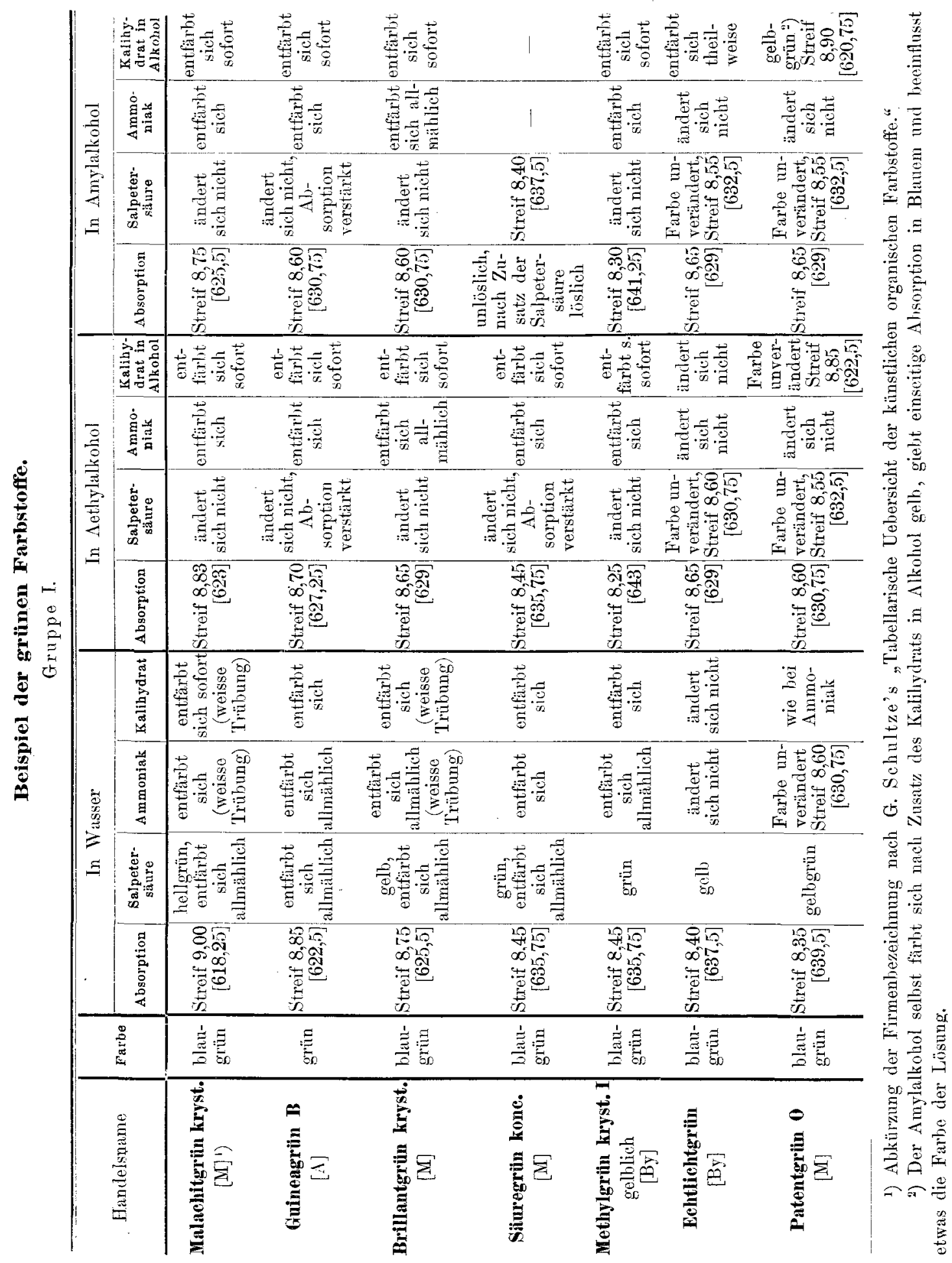




\begin{tabular}{|c|c|c|c|c|c|c|}
\hline \multirow{4}{*}{ 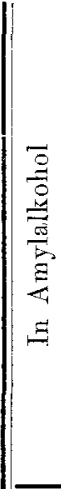 } & 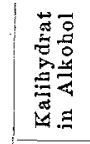 & 1 & 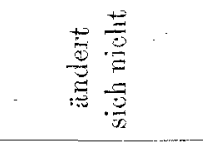 & 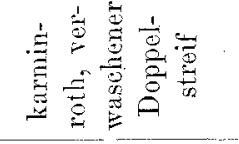 & 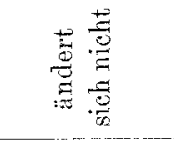 & 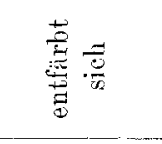 \\
\hline & $\frac{\dot{d}}{4}$ & 1 & 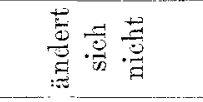 & 苞苞泀 & 莺离 & 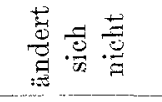 \\
\hline & 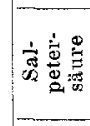 & 1 & 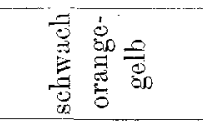 & 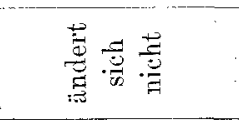 & 莺. & 莺 \\
\hline & 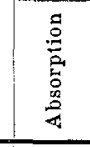 & 岕 & 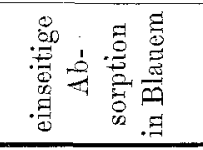 & 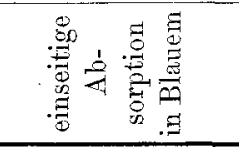 & 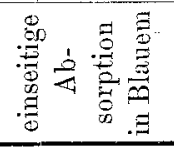 & 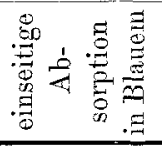 \\
\hline \multirow{4}{*}{ 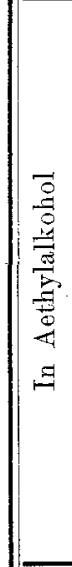 } & 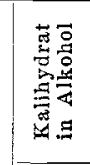 & 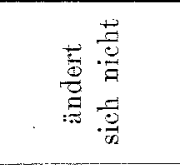 & 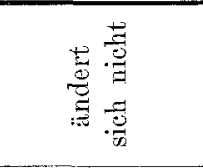 & 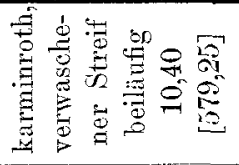 & 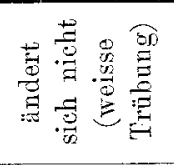 & 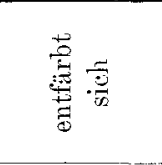 \\
\hline & 总: & 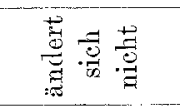 & 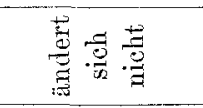 & 惫 & 常 & 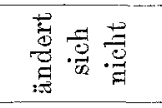 \\
\hline & 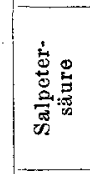 & 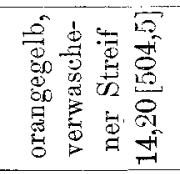 & 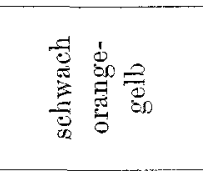 & 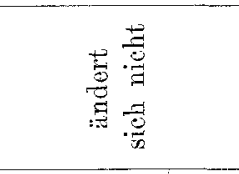 & 点 & 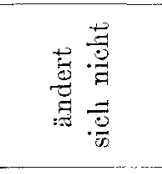 \\
\hline & 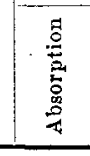 & 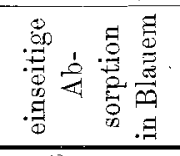 & 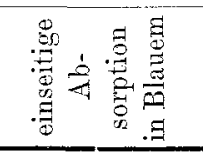 & 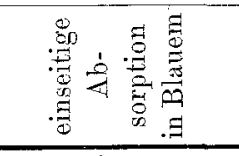 & 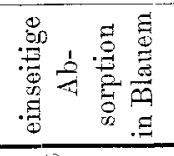 & 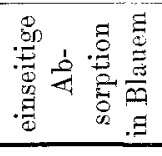 \\
\hline \multirow{4}{*}{ 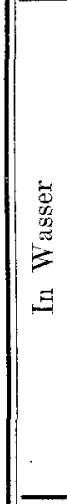 } & 票 & 苞 & 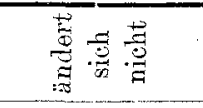 & 产. & 氖 & 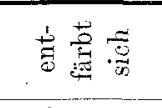 \\
\hline & 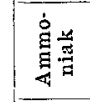 & 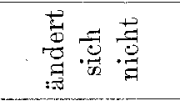 & 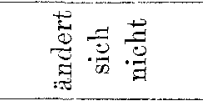 & 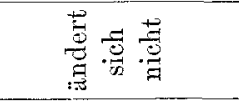 & 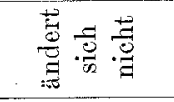 & 窇泀 \\
\hline & 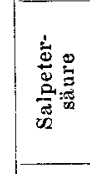 & 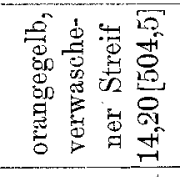 & 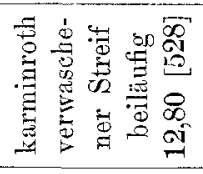 & 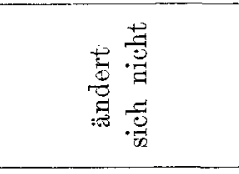 & 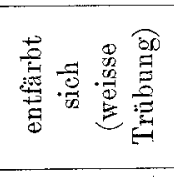 & 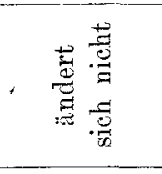 \\
\hline & $\begin{array}{l}\text { : } \\
\stackrel{0}{0} \\
0 \\
0 \\
0 \\
0\end{array}$ & 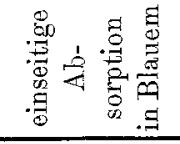 & 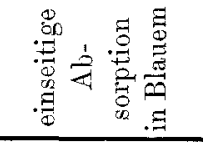 & 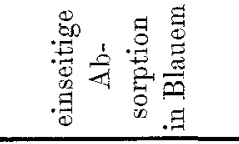 & 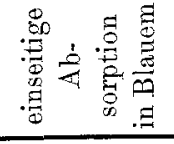 & 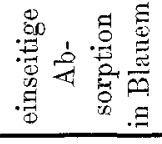 \\
\hline & 造 & $\hat{\vec{\partial}}$ & 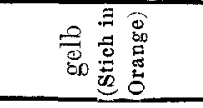 & $\stackrel{8}{8}$ & $\stackrel{\vec{B}}{\vec{D}}$ & $\stackrel{1}{0} \widehat{0}$ \\
\hline & 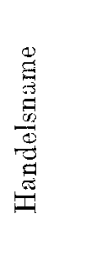 & 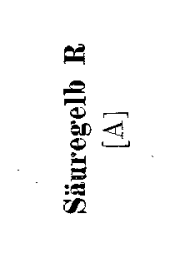 & 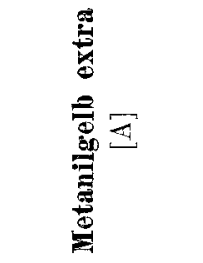 & 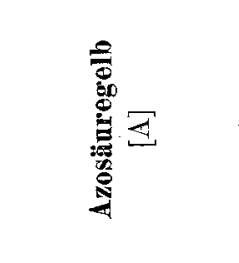 & 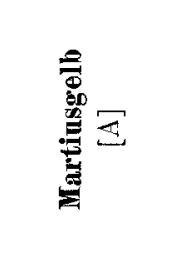 & 恶 \\
\hline
\end{tabular}


Beispiel der

Gruppe I.

\begin{tabular}{|c|c|c|c|c|c|}
\hline \multirow{2}{*}{ Handelsname } & \multirow{2}{*}{ Farbe } & \multicolumn{4}{|c|}{ In Wasser } \\
\hline & & Absorption & Salpetersäure & Ammoniak & Kalihydrat \\
\hline $\begin{array}{c}\text { Tiürkisblau } \\
{[\mathrm{By}]}\end{array}$ & blan & $\begin{array}{l}\text { Streif } 8,55 \\
{[\quad[632, \overline{0}]}\end{array}$ & $\begin{array}{l}\text { grünlich, } \\
\text { Absorption } \\
\text { unverändert }\end{array}$ & $\begin{array}{c}\text { ündert sich } \\
\text { nicht }\end{array}$ & $\begin{array}{c}\text { entfärbt sich } \\
\text { allmählich }\end{array}$ \\
\hline$\underset{[\mathrm{M}]}{\operatorname{Cyaniu}} \mathbf{B}$ & $\begin{array}{l}\text { blau- } \\
\text { grünlich }\end{array}$ & $\begin{array}{r}\text { Streif } 8,50 \\
{[634]}\end{array}$ & gelb & $\begin{array}{c}\text { Farbe } \\
\text { unverändert, } \\
\text { Streif } 8,80 \\
{[624]}\end{array}$ & $\begin{array}{c}\text { wie bei } \\
\text { Ammoniak }\end{array}$ \\
\hline
\end{tabular}

Gruppe II.

\begin{tabular}{|c|c|c|c|c|c|}
\hline $\begin{array}{c}\text { Methylviolett } 1 \text { B } \\
{[\mathrm{By}]}\end{array}$ & violett & $\begin{array}{r}\text { Hauptstreif } 10,05 \\
{[588,25]} \\
\text { Nebenstreif } 12,40 \\
{[535,5]}\end{array}$ & $\begin{array}{c}\text { blau, } \\
\text { dann grün, } \\
\text { Streif } 8,70 \\
{[627,20]}\end{array}$ & $\begin{array}{l}\text { ändert sich } \\
\text { nicht }\end{array}$ & $\begin{array}{l}\text { entfärbt sich } \\
\text { sofort }\end{array}$ \\
\hline $\begin{array}{c}\text { Methylviolett } 6 \mathbf{B} \\
{[\mathrm{By}]}\end{array}$ & violett & $\begin{array}{r}\text { Hauptstreif } 9,85 \\
{[\check{593,25]}} \\
\text { Nebenstreif } 12,20 \\
{[539,25]}\end{array}$ & $\begin{array}{c}\text { blau, } \\
\text { dann grün } \\
\text { Streif } 8,50 \\
{[631]}\end{array}$ & $\begin{array}{l}\text { entfärbt sich } \\
\text { theilweise }\end{array}$ & $\begin{array}{l}\text { entfärbt sich } \\
\text { allmählich }\end{array}$ \\
\hline
\end{tabular}

Gruppe III.

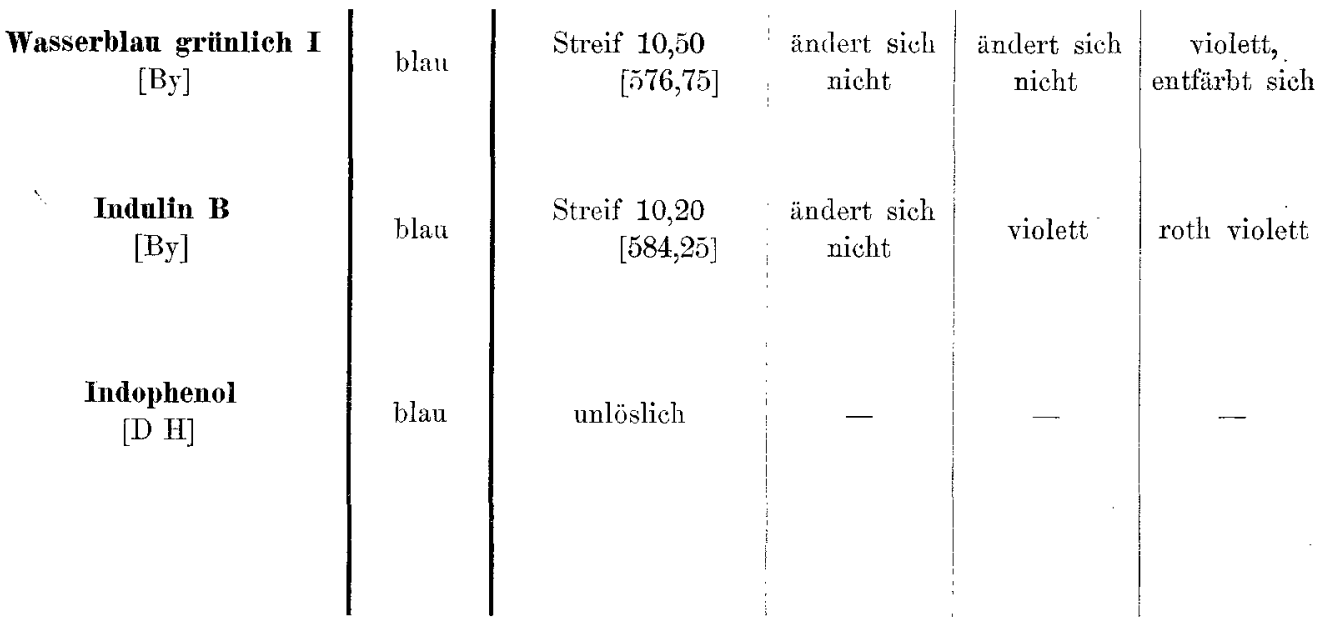


blauen Farbstoffe.

Gruppe I.

\begin{tabular}{|c|c|c|c|c|c|c|c|}
\hline \multicolumn{4}{|c|}{ In Aethylalkohol } & \multicolumn{4}{|c|}{ In Amylalkohol } \\
\hline Absorption & $\begin{array}{l}\text { Salpeter- } \\
\text { säure }\end{array}$ & Ammoniak & $\begin{array}{l}\text { Kalihydrat } \\
\text { in Alkohol }\end{array}$ & Absorption & Salpctersäure & $\underset{\text { niak }}{\text { Ammo- }}$ & $\begin{array}{l}\text { Kalihydrat } \\
\text { in Alkohol }\end{array}$ \\
\hline $\begin{array}{l}\text { Streif } 8,35 \\
{[639,5]}\end{array}$ & $\begin{array}{c}\text { ändert } \\
\text { sich nicht }\end{array}$ & $\begin{array}{c}\text { ändert } \\
\text { sich nicht }\end{array}$ & $\begin{array}{c}\text { entfärbt } \\
\text { sich }\end{array}$ & $\begin{array}{l}\text { Streif } 8,30 \\
\quad[641,25]\end{array}$ & $\begin{array}{l}\text { ändert sich } \\
\text { nicht }\end{array}$ & $\begin{array}{c}\text { ändert } \\
\text { sich nicht }\end{array}$ & $\begin{array}{l}\text { entfürbt } \\
\text { sich }\end{array}$ \\
\hline $\begin{array}{l}\text { Streif } 8,90 \\
{[620,75]}\end{array}$ & $\begin{array}{c}\text { ändert } \\
\text { sich nicht }\end{array}$ & $\begin{array}{c}\text { ändert } \\
\text { sich nicht }\end{array}$ & $\begin{array}{c}\text { entfärbt } \\
\text { sich } \\
\text { theilweise }\end{array}$ & $\begin{array}{r}\text { Streif } 8,80 \\
{[624]}\end{array}$ & $\begin{array}{c}\text { Farbe } \\
\text { unverändert, } \\
\text { Streif } 8,70 \\
{[627,25]}\end{array}$ & $\begin{array}{c}\text { ändert } \\
\text { sich nicht }\end{array}$ & $\begin{array}{c}\text { grün } \\
\text { (infolge } \\
\text { Gelbwer- } \\
\text { dens des } \\
\text { Amyl- } \\
\text { alkohols.) }\end{array}$ \\
\hline
\end{tabular}

Gruppe II.

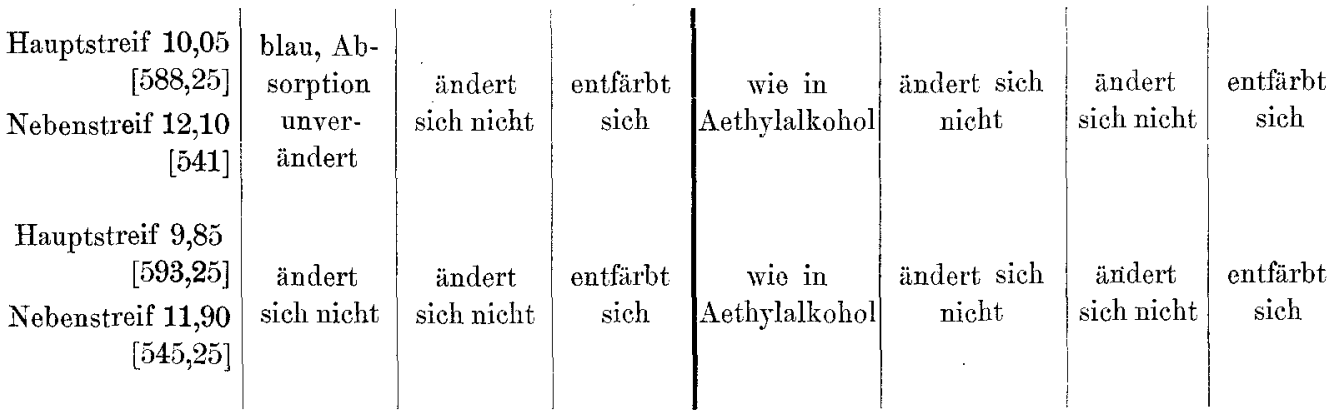

Gruppe III.

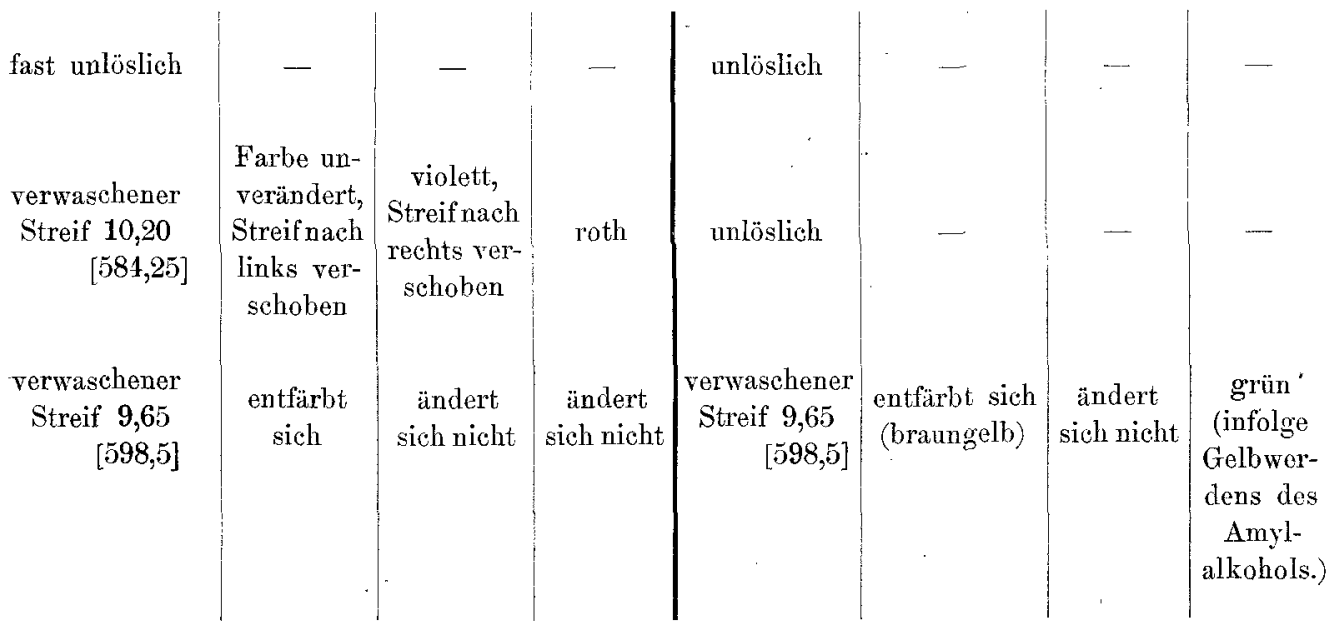

